\title{
RADIOTERAPIA LUMBOAÓRTICA EN PACIENTES CON CÁNCER DE CUELLO UTERINO. EXPERIENCIA DEL INSTITUTO NACIONAL DEL CÁNCER
}

\author{
Alejandro Santini B. ${ }^{1}$, Sergio Becerra S. ${ }^{1}$, Patricio Gayan P. ${ }^{2}$, Marcela Cárcamo I. ${ }^{3}$, \\ Benjamín Bianchi G. 4 \\ ${ }^{1}$ Radioterapeuta Oncólogo, ${ }^{2}$ Ginecólogo Oncólogo, ${ }^{3}$ Epidemióloga, ${ }^{4}$ Programa de Radioterapia, Instituto Nacional \\ del Cáncer.
}

\section{RESUMEN}

Antecedentes: El cáncer de cérvicouterino continúa siendo una enfermedad prevalente en Chile. Es frecuente recibir pacientes en etapas IIB y IIIB donde el riesgo de compromiso ganglionar pelviano y lumboaórtico es elevado y el tratamiento es radioquimioterapia. Objetivo: Análisis retrospectivo de pacientes con cáncer cérvicouterino que recibieron radioterapia que incluía los territorios lumboaórticos. Método: Revisión de pacientes con cáncer cérvicouterino tratadas entre 1995 y 2007 en el Instituto Nacional del Cáncer, Santiago, Chile. En 39 pacientes el tratamiento incluyó las cadenas lumboaórticas. Se analizó toxicidad y evolución. Se utilizaron dos técnicas de radioterapia: la primera durante la década del 90 , donde se empleaban dos campos paralelos y opuestos (anterior y posterior) y una segunda actual, donde se irradian en conjunto la pelvis y los lumboaórticos a través de 4 campos (laterales y AP-PA). Resultados: El análisis dosimétrico de las dos técnicas confirma un mayor volumen irradiado de tejidos normales con la técnica de dos campos, fundamentalmente intestino delgado. La toxicidad fue significativamente diferente, siendo la que utilizamos hoy menos tóxica y con bajas complicaciones gastrointestinales, a pesar de recibir un tratamiento de radio-quimioterapia concomitante ( $55 \%$ vs $0 \%)$. Conclusión: La radioterapia lumboaórtica es un tratamiento con una tolerancia aceptable incluso con quimioterapia concomitante. Esta revisión nos obliga a elaborar un nuevo trabajo para evaluar si la radioterapia lumboaórtica reduce el riesgo de recidiva y aumenta la sobrevida.

\section{PALABRAS CLAVE: Cáncer cérvico-uterino, radioterapia}

\section{SUMMARY}

Background: Uterine cancer is still a prevalent disease in Chile. Is common to treat patients with tumors in stages IIB and IIIB where the risk of pelvic and paraortic limph node involvement is very high. Its treatment is radio-chemotherapy. Objective: To present a retrospective analysis of patients that suffered cervix-uterine cancer who were treated with radiotherapy including the aortic-lumbar area. Methods: From the revision of patients who were treated of cervix-uterine cancer between the years 1995 and 2007, 39 were treated including aortic-lumbar chains. Evolution and toxicity were analyzed. Two radiotherapy techniques were used. The first one, during the nineties, included two parallel previous and later and opposed fields, and a second technique, currently used, where pelvis and paraortic are radiated at the same time through four lateral (AP-PA) fields. Results: The dosimeter analysis of both techniques shows that there is a higher volume of radiated normal tissue with the two fields techniques, mainly in the small bowel. On the other hand, the toxicity was significantly different being today's technique less toxic and showing low gastrointestinal 
side effects, despite receiving a simultaneous radio-chemotherapy ( $55 \%$ vs $0 \%$ ). Conclusion: The paraortic radiotherapy treatment has an acceptable level of tolerance even under simultaneous radio-chemotherapy. Given the results of this study, we see the need for undergoing a new research project in order to evaluate if the aortic-lumbar radiotherapy may reduce the risk of relapse and increase in survival rate.

\section{KEY WORDS: Uterine cervical carcinoma, radiotherapy}

\section{INTRODUCCIÓN}

El cáncer de cuello uterino en Chile continúa siendo una enfermedad prevalente (1) y es una de las localizaciones tumorales más frecuentes tratadas en el Instituto Nacional del Cáncer.

Desde hace más de 3 décadas, se discute sobre la indicación de la radioterapia (RT) a nivel lumboaórtico (LA), en el tratamiento del cáncer cérvico uterino. A lo largo de todos estos años se han realizado varias publicaciones que intentan arrojar una orientación al respecto (2-4). Uno de los aspectos más importantes que llevaron a esta discusión, es el hecho de que este tratamiento aumentaría las complicaciones, principalmente gastrointestinales. Es por este motivo que se ha intentado mejorar la técnica quirúrgica, abordando la región sin entrar al peritoneo y evitar el tratamiento de pacientes sin enfermedad a este nivel y mejorando la técnica de tratamiento radiante $(5,6)$.

En el Instituto Nacional del Cáncer (INC), desde hace 4 años no se emplea la técnica clásica de irradiación lumboaórtica, donde se hacían campos separados de los pélvicos y se emplea una técnica de 4 campos que incluyen la pelvis y los ganglios LA en forma conjunta. Esta técnica incluiría menos volumen de tejidos normales, fundamentalmente intestino, mejorando la tolerancia tanto aguda como crónica. La técnica anterior que se empleaba en la década de 1990, era una técnica secuencial, iniciando primero por la pelvis y posteriormente la región lumboaórtica, siendo esta ultima tratada por dos campos AP-PA. Debido a esta situación, planteamos como objetivo comparar dosimétricamente estas dos técnicas de tratamiento y evaluar la tolerancia de ambas.

\section{MATERIAL Y MÉTODOS}

Este trabajo es el análisis de una serie de casos y la comparación de dos técnicas de tratamiento de radioterapia. Se revisaron las fichas de todas las pacientes portadoras de cáncer de cuello uterino atendidas en el INC, Santiago de Chile, desde enero de 1995 a marzo de 2007. Se analizaron las planillas o cartolas de tratamiento de radioterapia y se rescataron las pacientes que recibieron tratamiento de radioterapia a nivel LA. Como criterios de inclusión se consideraron a todas aquellas pacientes que recibieron irradiación a dicho nivel. Se excluyeron dos pacientes en quienes si bien se realizó tratamiento a nivel $\mathrm{LA}$, el objetivo del mismo fue paliativo y no curativo, así como una paciente que recibió $\mathrm{RT}$ LA por una recidiva a dicho nivel luego de un tratamiento exclusivamente pelviano. En ellas se analizó la técnica de tratamiento (dosis, campos y volúmenes) así como la tolerancia aguda y crónica de los tratamientos.

Cuando se analizó la técnica de tratamiento, se pudo dividir la población en dos grupos, de acuerdo al tratamiento recibido:

Primer grupo (12 pacientes): aquellas en quienes se empleó una técnica que incluía campos separados, por un lado la pelvis con 4 campos (técnica de caja), y por otro lado la región LA con dos campos paralelos y opuestos AP-PA. Entre ellos 8 pacientes se trataron con cobaltoterapia y 4 con acelerador lineal. Estas dos etapas de tratamiento se hacían en forma sucesiva, primero la pelvis y posteriormente la región lumboaórtica. La planificación fue en $2 \mathrm{D}$ para 8 de las 12 pacientes y en las 4 restantes fue 3D. Los límites de los campos lumboaórticos se ubicaron, el superior a nivel de T10 y el inferior a nivel del borde superior del campo pelviano (L4-L5). Los bordes laterales se colocaron arbitrariamente a nivel del borde lateral de las apófisis transversas de las vértebras, teniendo un ancho aproximado de 10 $\mathrm{cm}$. La dosis que se entregó fue de $45 \mathrm{~Gy}$ en 25 fracciones iguales de 1,8 Gy 5 veces a la semana. En este primer grupo de pacientes, solo 2 de las 12 pacientes recibieron tratamiento de quimioterapia concomitante. El tiempo promedio total de tratamiento fue de 13,5 semanas. En estas pacientes se empleo braquiterapia con baja tasa de dosis (LDR) una vez terminada la radioterapia externa.

Segundo grupo (27 pacientes): se empleo una técnica más moderna, con campos únicos que incluían la pelvis y la región LA en forma conjunta; en ellas todo el tratamiento se realizó con 4 campos AP-PA y laterales. La planificación se realizó forma conformada 3D para todos los casos. En este grupo de tratamiento 24 de 27 (88\%) recibieron un tra- 
tamiento combinado de radioterapia y quimioterapia en forma concomitante. En estas pacientes se empleó cisplatino $40 \mathrm{mg} / \mathrm{m}^{2}$ en forma semanal y se utilizaron entre 5 y 6 ciclos. El tiempo promedio de la totalidad del tratamiento fue de 8,8 semanas. En las pacientes de este segundo grupo, la braquiterapia fue de alta tasa en 23 de ellas (entre 3 y 5 fracciones de 5,5 a 7,5 Gy por fracción), mientras que en 3 fue de LDR, y una paciente fue tratada exclusivamente con radioterapia externa.

En ambos grupos se realizó un boost o sobreimpresión a nivel de uno o dos parametrios de entre 10 y 14 Gy cuando los mismos se encontraban comprometidos.

Utilizando las herramientas del sistema de planificación que empleamos en la actualidad (ECLIPSE 8.5), se compararon las dos técnicas de tratamiento, las curvas de isodosis y los histogramas dosis volumen (HDV). EI HDV es una herramienta muy importante en los sistemas de planificación modernos en 3 dimensiones (3D), ellos permiten cuantificar el volumen de un determinado órgano que recibe un nivel de dosis elegido (7). Existe una correlación importante entre los volúmenes de dosis y los efectos sobre determinado tejido y es por eso que la comparación de los HDV es importante a la hora de comparar dos tratamientos diferentes (7-9).

Para dicho análisis como ejemplo elegimos al azar un caso en particular y simulamos las dos técnicas de tratamiento, analizando los HDV de cada uno, es decir en la misma paciente se realizó una planificación virtual de la dos técnicas empleadas. Con el objetivo de analizar las complicaciones se clasificó la tolerancia de acuerdo a la escala RTOG (10) (Tabla I). Se estableció un nivel de confianza del $95 \%$ y el programa estadístico empleado fue STATA 10.0 .

\section{RESULTADOS}

En la Tabla II se describen las características generales de la población en estudio.

Análisis dosimétrico: Como se aprecia en las Figuras 1 y 2 (que muestran la curva de isodosis del $100 \%$ de la dosis prescrita), se encontró una gran diferencia en el volumen de tejido abdominal incluido con las dos técnicas de tratamiento. El volumen incluido dentro del $100 \%$ de la dosis prescrita es casi el doble con la técnica del grupo 1 respecto a la del grupo $2\left(152 \mathrm{~cm}^{3}\right.$ vs $\left.282 \mathrm{~cm}^{3}\right)$.

En la Figura 3 se analizan los HDV de las dos técnicas de tratamiento. En ellos se aprecia clara- mente que la dosis máxima a nivel intestinal (puntos calientes) es casi 10 Gy mayor con la técnica de campos AP-PA respecto a la de 4 campos $(61,1$ Gy vs 51,3 Gy respectivamente). La dosis, máxima o los puntos calientes también se correlacionan con el riesgo de complicaciones. A nivel pelviano, dado que la técnica aquí es similar el grupo 1 y 2 no hay diferencias en los volúmenes de tejido normal irradiado. O sea que la diferencia dosimétrica fundamental entre las dos técnicas esta a nivel abdominal y no pelviana.

Análisis de toxicidad: El análisis de la toxicidad se realizó de acuerdo a la escala de la RTOG (11) (en un grupo de pacientes, las tratadas a finales de 2006 y durante el 2007 no se pudo analizar la toxicidad crónica por el corto seguimiento de las mismas), y los resultados tanto agudos como crónicos se desarrolla en las Tablas III y IV.

Cuando analizamos por separado la toxicidad aguda y crónica según la técnica de tratamiento, se obtuvo un menor porcentaje de efectos agudos y crónicos gastrointestinales, aunque solamente para los efectos crónicos la diferencia fue estadísticamente significativa $(p=0,003)$. Estos resultados concuerdan con el análisis dosimétrico que muestra un mayor volumen de tejido intestinal irradiado.

No hubo diferencia en la toxicidad genitourinaria tanto aguda como crónica, ni en la toxicidad hematológica (Tablas V-IX). Esto se correlaciona bien con el análisis dosimétrico antes mencionado, donde se apreciaba una mayor cantidad de intestino incluido en la técnica de dos campos y no una diferencia en el volumen vesical irradiado con cada una de las técnicas. La distribución de dosis e la pelvis es similar para las dos técnicas.

Estos resultados respecto a la toxicidad se ven a pesar de que las pacientes del grupo 2 (4 campos) recibieron quimioterapia concomitante en el $88 \%$ de los casos (24 de 27 pacientes), a diferencia de las pacientes con la técnica de dos campos paralelos en que fue solo del $16 \%$ (2 de 12 ).

El tiempo medio total de duración del tratamiento en las pacientes el grupo 1 fue de 13,5 semanas (10-16 semanas) comparado con las del grupo 2 donde fue de 8,8 semanas (6-14,4 semanas).

\section{DISCUSIÓN}

En base a los resultados de este estudio, podemos decir que en el análisis dosimétrico de las diferentes técnicas de tratamiento, los volúmenes irradiados se correlacionarían directamente con la tolerancia y la toxicidad de los mismos. 
Tabla I

CRITERIOS DE ETAPIFICACIÓN PARA TOLERANCIA Y EFECTOS SECUNDARIOS DE LA RTOG (11)

Grados de toxicidad

\begin{tabular}{|c|c|c|c|c|c|}
\hline \multicolumn{6}{|c|}{ Grados de toxicidad } \\
\hline & 0 & 1 & 2 & 3 & 4 \\
\hline $\begin{array}{l}\text { Toxicidad } \\
\text { gastrointestinal } \\
\text { aguda }\end{array}$ & $\begin{array}{c}\text { Sin } \\
\text { cambio }\end{array}$ & $\begin{array}{l}\text { Baja de peso } 5 \% \text {, } \\
\text { náusea, dolor o } \\
\text { diarrea que no } \\
\text { requiere } \\
\text { medicamento }\end{array}$ & $\begin{array}{l}\text { Baja de peso } 15 \% \text {, } \\
\text { dolor, náusea o } \\
\text { diarrea que requiere } \\
\text { medicamento }\end{array}$ & $\begin{array}{l}\text { Diarrea, } \\
\text { náuseas, que } \\
\text { requiere apoyo } \\
\text { parenteral, } \\
\text { hospitalización } \\
\text { o hemorragia }\end{array}$ & $\begin{array}{l}\text { Obstrucción } \\
\text { intestinal, } \\
\text { perforación, } \\
\text { hemorragia que } \\
\text { requiere } \\
\text { transfusión }\end{array}$ \\
\hline $\begin{array}{l}\text { Toxicidad } \\
\text { genitourinaria } \\
\text { aguda } \\
\\
\text { Toxicidad } \\
\text { hematológica } \\
\text { aguda }\end{array}$ & $\begin{array}{c}\text { Sin } \\
\text { cambio }\end{array}$ & $\begin{array}{l}\text { Disuria o } \\
\text { urgencia que } \\
\text { no requiere } \\
\text { medicamento }\end{array}$ & $\begin{array}{l}\text { Frecuencia } \\
\text { o nicturia } \\
\text { aumentada, } \\
\text { pero menos de } \\
\text { cada } 1 \text { hora. } \\
\text { Requiere } \\
\text { medicamento }\end{array}$ & $\begin{array}{l}\text { Frecuencia } \\
\text { o nicturia } \\
\text { cada } 1 \text { hora. } \\
\text { Dolor pélvico } \\
\text { que requiere } \\
\text { analgésicos } \\
\text { mayores o } \\
\text { hematuria }\end{array}$ & $\begin{array}{l}\text { Hematuria que } \\
\text { requiere } \\
\text { transfusión, } \\
\text { obstrucción } \\
\text { aguda, } \\
\text { ulceración } \\
\text { o necrosis }\end{array}$ \\
\hline $\begin{array}{l}\text { - GB (x 1000) } \\
\text { - PL (x 100 mil) } \\
\text { - NT (x 100) } \\
\text { - Hb (g/dl) } \\
\text { - Hcto (\%) }\end{array}$ & $\begin{array}{l}>4,0 \\
>100 \\
>1,9 \\
>11 \\
>32\end{array}$ & $\begin{array}{l}3,0-4,0 \\
73-100 \\
1,5-1,9 \\
9,5-11 \\
28-32\end{array}$ & $\begin{array}{l}2-3,2 \\
50-73 \\
1,0-1,5 \\
9,5-7,5 \\
<28\end{array}$ & $\begin{array}{l}1,0-2,0 \\
25-50 \\
0,5-1,0 \\
7,5-5,0 \\
\text { Requiere } \\
\text { transfusión }\end{array}$ & $\begin{array}{l}<1,0 \\
<25 \text { o hemorragia } \\
<0,5 \text { o sepsis }\end{array}$ \\
\hline $\begin{array}{l}\text { Toxicidad } \\
\text { gastrointestinal } \\
\text { crónica }\end{array}$ & $\begin{array}{c}\text { Sin } \\
\text { cambio }\end{array}$ & & & & \\
\hline $\begin{array}{l}\text { Toxicidad } \\
\text { genitourinaria } \\
\text { crónica }\end{array}$ & $\begin{array}{c}\text { Sin } \\
\text { cambio }\end{array}$ & $\begin{array}{l}\text { Ligera atrofia } \\
\text { epitelial, alguna } \\
\text { telangectasia, } \\
\text { hematuria } \\
\text { microscópica }\end{array}$ & $\begin{array}{l}\text { Aumento } \\
\text { moderado de la } \\
\text { frecuencia } \\
\text { miccional, } \\
\text { telangiectasia } \\
\text { moderada, } \\
\text { hematuria } \\
\text { macroscópica } \\
\text { ocasional }\end{array}$ & $\begin{array}{l}\text { Severa disuria y } \\
\text { aumento de la } \\
\text { frecuencia, } \\
\text { hematuria } \\
\text { frecuente, } \\
\text { reducción del } \\
\text { volumen menor } \\
\text { de } 150 \text { cc }\end{array}$ & $\begin{array}{l}\text { Necrosis vesical, } \\
\text { vejiga atrófica, } \\
\text { capacidad menor } \\
\text { a } 100 \mathrm{cc} \text {, cistitis } \\
\text { hemorrágica }\end{array}$ \\
\hline $\begin{array}{l}\text { Toxicidad } \\
\text { hematologica } \\
\text { crónica }\end{array}$ & $\begin{array}{c}\text { Sin } \\
\text { cambio }\end{array}$ & $\begin{array}{l}\text { Diarrea leve, } \\
\text { menos de } 5 \\
\text { veces al día, } \\
\text { rectorragia } \\
\text { ocasional }\end{array}$ & $\begin{array}{l}\text { Diarrea } \\
\text { moderada mas } \\
\text { de } 5 \text { veces al } \\
\text { día, cólicos } \\
\text { ocasionales, } \\
\text { secreción }\end{array}$ & $\begin{array}{l}\text { Obstrucción o } \\
\text { sangrado que } \\
\text { requiere } \\
\text { intervención } \\
\text { quirúrgica }\end{array}$ & $\begin{array}{l}\text { Necrosis, } \\
\text { perforación } \\
\text { o fístula }\end{array}$ \\
\hline $\begin{array}{l}\text { Toxicidad } \\
\text { hematológica } \\
\text { crónica }\end{array}$ & & & $\begin{array}{l}\text { mucosa y } \\
\text { rectorragia } \\
\text { frecuente }\end{array}$ & & \\
\hline $\begin{array}{l}\text { - GB (x 1000) } \\
\text { - PL (x 100 mil) } \\
\text { - NT (x 100) } \\
\text { - Hb (g/dl) } \\
\text { - Hcto (\%) }\end{array}$ & $\begin{array}{l}>4,0 \\
>100 \\
>1,9 \\
>11 \\
>32\end{array}$ & $\begin{array}{l}3,0-4,0 \\
73-100 \\
1,5-1,9 \\
9,5-11 \\
28-32\end{array}$ & $\begin{array}{l}2-3.2 \\
50-73 \\
1,0-1,5 \\
9,5-7,5 \\
<28\end{array}$ & $\begin{array}{l}1,0-2,0 \\
25-50 \\
0,5-1,0 \\
7,5-5,0 \\
\text { Requiere } \\
\text { transfusión }\end{array}$ & $\begin{array}{l}<1,0 \\
<25 \text { o hemorragia } \\
<0,5 \text { o sepsis }\end{array}$ \\
\hline
\end{tabular}


Tabla II

CARACTERÍSTICAS DE LA POBLACIÓN DE PACIENTES QUE RECIBIERON RADIOTERAPIA LUMBOAÓRTICA ENTRE 1995-2007

\begin{tabular}{|c|c|c|}
\hline $\begin{array}{l}\text { Número de } \\
\text { pacientes }\end{array}$ & 39 & \\
\hline Edad & $\begin{array}{l}\text { Promedio: } \\
47 \text { años }\end{array}$ & $\begin{array}{c}\text { Rango: } \\
\text { 27-78 años }\end{array}$ \\
\hline Etapa (FIGO) & $\begin{array}{l}\text { IB } \\
\text { IIA } \\
\text { IIB } \\
\text { IIIB } \\
\text { IV }\end{array}$ & $\begin{array}{l}12,5 \% \\
2,5 \% \\
53,8 \% \\
25,6 \% \\
2,5 \%\end{array}$ \\
\hline Hidronefrosis & 1 paciente & $2,5 \%$ \\
\hline Histología (n) & $\begin{array}{l}\text { Adenocarcinoma } \\
\text { Escamoso } \\
\text { Células pequeñas } \\
\text { Células claras }\end{array}$ & $\begin{array}{l}10 \%(4) \\
83 \%(33) \\
2,5 \%(1) \\
2,5 \%(1)\end{array}$ \\
\hline $\begin{array}{l}\text { Adenopatías } \\
\text { pelvianas }\end{array}$ & $\begin{array}{l}\mathrm{N}+\text { en TAC } \\
\text { Biopsia N+ } \\
\text { RMN N+ }\end{array}$ & $\begin{array}{l}43 \%(16) \\
10 \%(4) \\
2,5 \%(1)\end{array}$ \\
\hline Adenopatías LA & $\begin{array}{l}\mathrm{N}+\text { en } \mathrm{TAC} \\
\mathrm{N}+\text { en } \mathrm{RMN}\end{array}$ & $\begin{array}{l}25 \%(10) \\
2,5 \%(1)\end{array}$ \\
\hline Tratamiento & $\begin{array}{l}\text { Cirugía } \rightarrow \text { RT } \\
\text { RT }\end{array}$ & $\begin{array}{l}21 \% \\
79 \%\end{array}$ \\
\hline Equipo & $\begin{array}{l}\mathrm{AL} \\
\text { Cobalto }\end{array}$ & $\begin{array}{l}79 \% \\
21 \%\end{array}$ \\
\hline Grupo 1 & $\begin{array}{l}\text { Técnica } \\
\text { LA AP-PA }\end{array}$ & 12 pacientes \\
\hline Grupo 2 & $\begin{array}{l}\text { Técnica LA } 4 \text { campos } \\
\text { AP-PA + Laterales }\end{array}$ & 27 pacientes \\
\hline $\begin{array}{l}\text { Quimioterapia } \\
\text { concomitante }\end{array}$ & $\begin{array}{l}\text { Grupo } 1 \\
\text { Grupo } 2\end{array}$ & $\begin{array}{l}2 \text { pacientes } \\
24 \text { pacientes }\end{array}$ \\
\hline $\begin{array}{l}\text { Tiempo promedio } \\
\text { de tratamiento }\end{array}$ & $\begin{array}{l}\text { Grupo } 1 \\
\text { Grupo } 2\end{array}$ & $\begin{array}{l}13,5 \text { semanas } \\
8,8 \text { semanas }\end{array}$ \\
\hline
\end{tabular}

$\mathrm{N}+$ : ganglios palpables o visibles en TAC.

Durante muchos años se empleó en el Servicio de Radioterapia del INC, una técnica de tratamiento para la irradiación LA con dos campos paralelos y opuestos, anterior y posterior; incluso con equipo de cobaltoterapia. A partir de 2004, como consecuencia de la llegada de los sistemas de planificación en 3D, se comenzó a emplear una técnica más moderna de tratamiento, utilizando 4 campos conformados que incluyen tanto la pelvis como la región LA y todos los pacientes se trataron con energías iguales o mayores a $6 \mathrm{mev}$. Con la primera técnica ( 2 campos AP-PA) se irradia una mayor cantidad

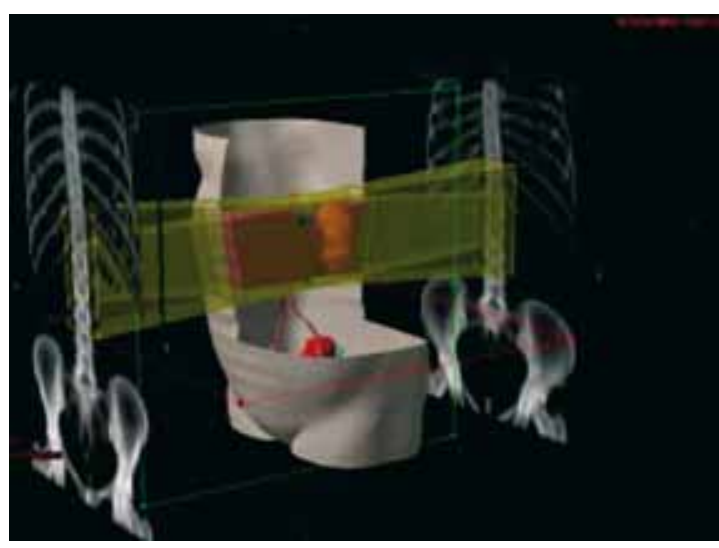

Figura 1. Técnica de tratamiento RT para la región LA con campos paralelos y opuestos AP-PA. Nótese en morado la curva de isodosis del $100 \%$ que incluye gran cantidad de tejido abdominal. En esta figura no esta representada la RT pelviana.

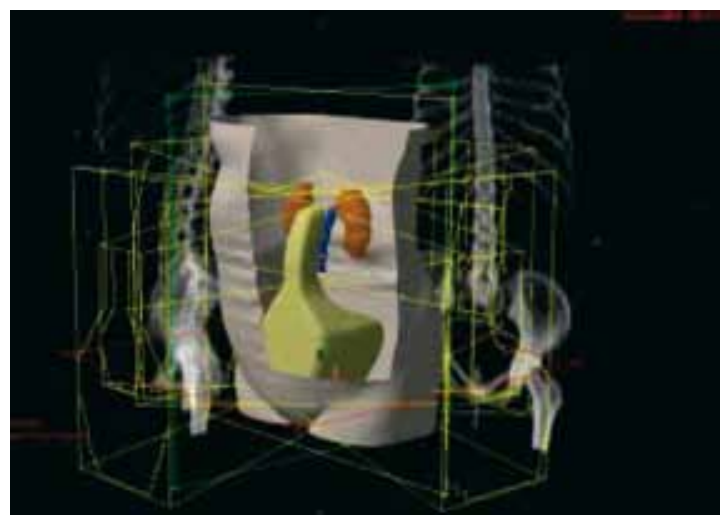

Figura 2. Técnica de tratamiento RT que incluye en un mismo campo de tratamiento la pelvis y LA. Nótese en amarillo el volumen de irradiación LA francamente menor que el volumen de irradiación pelviana.

de tejidos normales, fundamentalmente un gran volumen de intestino delgado, no así de vejiga (Figura 1 y 2). Como ejemplo de ello, se analizaron los HDV de las dos técnicas (Figura 3-5). En dicho análisis, se pudo observar no sólo un aumento del volumen de intestino irradiado en casi el $100 \%(152 \mathrm{~cm} 3$ vs $282 \mathrm{~cm}^{3}$ ), sino un aumento en la dosis máxima recibida a dicho nivel (51,3 Gy vs 61,1 Gy). Tanto la dosis máxima, como el volumen total de intestino irradiado, son parámetros importantes y directamente correlacionados con la toxicidad. Recordemos que el riesgo de complicaciones depende en gran medida del volumen de tejido irradiado. 


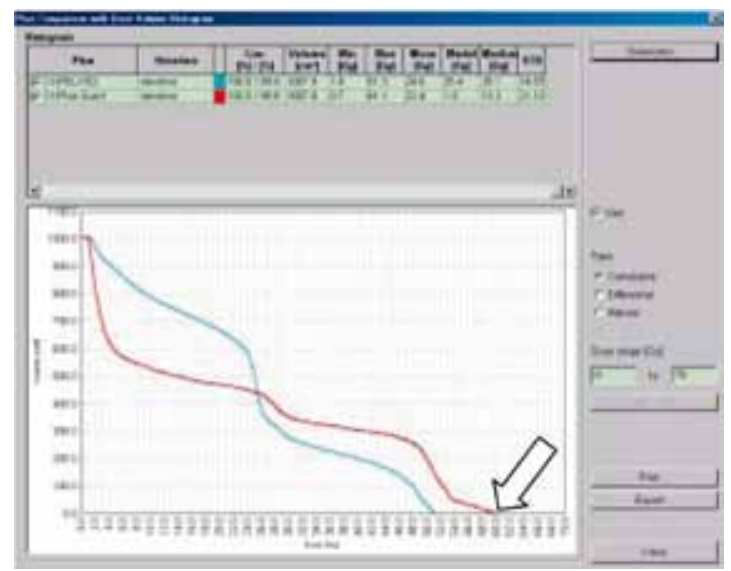

Figura 3. HDV que compara los volumen de intestino incluidos en las dos técnicas de tratamiento. En rojo la técnica de campos AP-PA y en azul la de 4 campos. Nótese la dosis máxima de 61,1 Gy vs 51,3 Gy respectivamente (flecha).

Tabla III

TOXICIDAD AGUDA GASTROINTESTINAL (GI), GENITOURINARIA (GU) Y HEMATOLÓGICA (H) EN TODA LA POBLACIÓN ESTUDIADA

\begin{tabular}{cccr}
\hline $\begin{array}{l}\text { Grado de toxicidad } \\
\text { (escala RTOG) }\end{array}$ & $\begin{array}{c}\mathrm{n}(\%) \\
\mathrm{Gl}\end{array}$ & $\begin{array}{c}\mathrm{n}(\%) \\
\mathrm{GU}\end{array}$ & $\begin{array}{c}\mathrm{n}(\%) \\
\mathrm{H}\end{array}$ \\
\hline 0 & $4(10)$ & $9(23)$ & $23(58)$ \\
1 & $18(46)$ & $21(55)$ & $9(23)$ \\
2 & $15(38)$ & $6(15)$ & $6(15)$ \\
3 & $2(5)$ & $2(5)$ & $2(5)^{\star}$ \\
4 & $0(0)$ & $0(0)$ & $0(0)$ \\
\hline
\end{tabular}

Ninguna paciente suspendió tratamiento por toxicidad aguda gastrointestinal. *Anemia que requirió transfusión en 2 oportunidades.

Tabla IV

TOXICIDAD CRÓNICA GASTROINTESTINAL

(GI) Y GENITOURINARIA (GU) EN 28 PACIENTES*

\begin{tabular}{ccc}
\hline $\begin{array}{l}\text { Grado de toxicidad } \\
\text { (escala RTOG) }\end{array}$ & $\begin{array}{c}\mathrm{n}(\%) \\
\mathrm{Gl}\end{array}$ & $\begin{array}{c}\mathrm{n}(\%) \\
\mathrm{GU}\end{array}$ \\
\hline 0 & $20(71)$ & $24(88)$ \\
1 & $3(10)$ & $4(12)$ \\
2 & $4(14)$ & $0(0)$ \\
3 & $1(3)$ & $0(0)$ \\
4 & $0(0)$ & $0(0)$ \\
\hline
\end{tabular}

*12 pacientes no tienen seguimiento para evaluar toxicidad crónica por ser tratadas durante el 2007
Tabla V

\section{TOXICIDAD AGUDA GASTROINTESTINAL SEGÚN LA TÉCNICA DE TRATAMIENTO DE RADIOTERAPIA*}

\begin{tabular}{ccc}
$\begin{array}{l}\text { Grado de toxicidad } \otimes \\
\text { (escala RTOG) }\end{array}$ & $\begin{array}{c}\text { Técnica de campos } \otimes \\
\text { paralelos y opuestos } \otimes \\
\text { (AP-PA) }\end{array}$ & 4 campos \\
\hline 0 & $8 \%$ & $11 \%$ \\
1 & $50 \%$ & $44 \%$ \\
2 & $41 \%$ & $37 \%$ \\
3 & $1 \%$ & 0 \\
4 & 0 & 0
\end{tabular}

* diferencia no significativa

\section{Tabla VI}

\section{TOXICIDAD AGUDA GENITOURINARIA SEGÚN LA TÉCNICA DE TRATAMIENTO DE RADIOTERAPIA*}

\begin{tabular}{ccc}
\hline $\begin{array}{l}\text { Grado de toxicidad } \\
\text { (escala RTOG) }\end{array}$ & $\begin{array}{c}\text { Técnica de campos } \\
\text { paralelos y opuestos } \\
\text { (AP-PA) }\end{array}$ & 4 campos \\
\hline 0 & $27 \%$ & $22 \%$ \\
1 & $27 \%$ & $66 \%$ \\
2 & $27 \%$ & $11 \%$ \\
3 & $18 \%$ & 0 \\
4 & 0 & 0 \\
\hline
\end{tabular}

* diferencia no significativa

Tabla VII

TOXICIDAD AGUDA HEMATOLÓGICA SEGÚN LA TÉCNICA DE TRATAMIENTO DE RADIOTERAPIA*

\begin{tabular}{ccc}
\hline $\begin{array}{l}\text { Grado de toxicidad } \\
\text { (escala RTOG) }\end{array}$ & $\begin{array}{c}\text { Técnica de campos } \\
\text { paralelos y opuestos } \\
\text { (AP-PA) }\end{array}$ & 4 campos \\
\hline 0 & $66 \%$ & $55 \%$ \\
1 & $16 \%$ & $25 \%$ \\
2 & $16 \%$ & $11 \%$ \\
3 & 0 & $3 \%$ \\
4 & 0 & 0 \\
\hline
\end{tabular}

* diferencia no significativa 
Tabla VIII

\section{TOXICIDAD CRÓNICA GASTROINTESTINAL SEGÚN LA TÉCNICA DE TRATAMIENTO DE RADIOTERAPIA*}

\begin{tabular}{ccc}
\hline $\begin{array}{l}\text { Grado de toxicidad } \\
\text { (escala RTOG) }\end{array}$ & $\begin{array}{c}\text { Técnica de campos } \\
\text { paralelos y opuestos } \\
\text { (AP-PA) }\end{array}$ & 4 campos \\
\hline 0 & $44 \%$ & $84 \%$ \\
1 & 0 & $15 \%$ \\
2 & $44 \%$ & 0 \\
3 & $11 \%$ & 0 \\
4 & 0 & 0 \\
\hline
\end{tabular}

${ }^{*} p=0,003$

\section{Tabla IX}

TOXICIDAD CRÓNICA GENITOURINARIA SEGÚN LA TÉCNICA DE TRATAMIENTO DE RADIOTERAPIA*

\begin{tabular}{ccc}
\hline $\begin{array}{l}\text { Grado de toxicidad } \\
\text { (escala RTOG) }\end{array}$ & $\begin{array}{c}\text { Técnica de campos } \\
\text { paralelos y opuestos } \\
\text { (AP-PA) }\end{array}$ & 4 campos \\
\hline 0 & $75 \%$ & $95 \%$ \\
1 & $25 \%$ & $5 \%$ \\
2 & 0 & 0 \\
3 & 0 & 0 \\
4 & 0 & 0 \\
\hline
\end{tabular}

* diferencia no significativa

Dicho hecho, pudo reflejarse en una menor toxicidad gastrointestinal crónica con las técnicas más modernas $(0 \%$ de toxicidad grado 2 y 3 versus $55 \%, p=0,003)$. Esta mejoría en la calidad de los tratamientos hace que los mismos tengan una baja tasa de complicaciones, incluso con el empleo de quimioterapia concomitante, que constituye el tratamiento estándar en nuestro hospital. Esta buena tolerancia también se ve reflejada indirectamente cuando analizamos el tiempo total de tratamiento. En este sentido, el tiempo promedio de duración del tratamiento para el grupo 1 fue de 13,5 semanas comparado con 8,8 semanas del grupo 2 . Esta prolongación en el tiempo total del tratamiento tiene una explicación en el hecho de que el tratamiento en las pacientes del grupo 1 es secuencial, primero se trataba la pelvis y luego la región lumboaórtica, mientras que en la técnica empleada en el grupo 2 es concomitante, o sea se tratan las dos regiones en el mismo momento. Sin embargo, el mantener el tiempo total de tratamiento en 8 semanas podría también ser el reflejo de un tratamiento bien tolerado, acorde al registro de toxicidad aguda.

Si bien el objetivo de este análisis no es evaluar

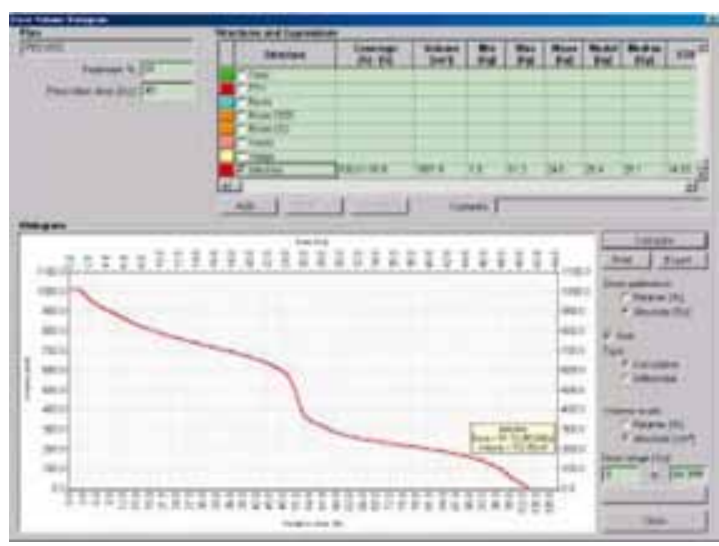

Figura 4. HDV de intestino con técnica de 4 campos. Volumen de irradiado con el $100 \%$ de la dosis $152 \mathrm{~cm}^{3}$.

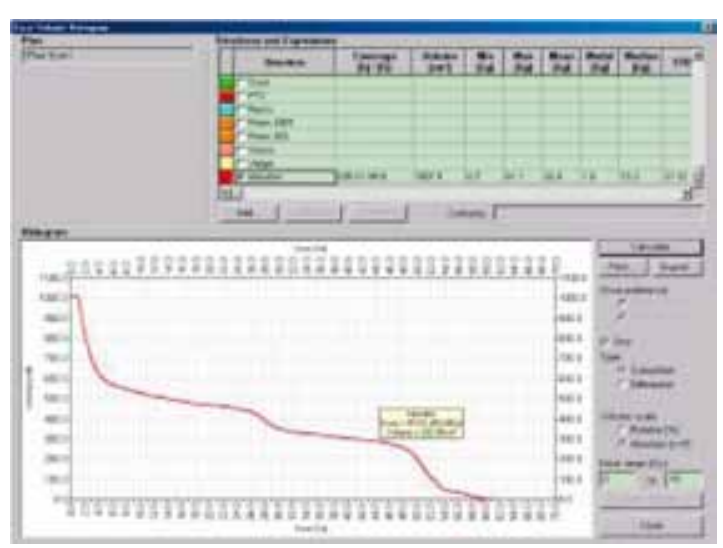

Figura 5. HDV de intestino con técnica de 4 campos. Volumen de irradiado con el $100 \%$ de la dosis $282 \mathrm{~cm}^{3}$.

los resultados terapéuticos de dicho tratamiento, cabe suponer que un tratamiento completado en 8,8 semanas tendría un mejor resultado comparado con otro que demore más de 13 semanas. El cáncer cervicouterino es uno de los tumores con mayor dependencia del tiempo total del tratamiento por lo que la mayoría de los centros recomiendan completarlo al menos en 9 semanas (11).

\section{CONCLUSIÓN}

En nuestra experiencia, el tratamiento de radioterapia que incluye las regiones lumboaórticas, es un tratamiento que con las técnicas modernas de irradiación no tiene tasas de toxicidad elevadas. Es por lo tanto un tratamiento realizable y tolerable a pesar de que en la actualidad dichos tratamientos se hacen habitualmente en concomitancia con quimioterapia 
(cisplatino mono droga semanal). En la serie analizada se confirma la factibilidad de dicho tratamiento. El análisis dosimétrico de las técnicas de tratamiento nos permite explicar esta buena tolerancia, dado que se aprecia un menor volumen de tejido normal irradiado así como puntos calientes (puntos de dosis altas) menos intensos.

Estos resultados nos obligan a desarrollar un trabajo prospectivo, randomizado para comparar no sólo la tolerancia del tratamiento, sino la efectividad o eficiencia terapéutica y de esta manera poder pautar una selección de tratamientos mas adecuada a cada situación clínica.

\section{BIBLIOGRAFÍA}

1. Donoso E, Cuello M, Villarroel L. Reducción de la mortalidad por cáncer de cuello uterino en Chile, 19902003. Rev Chil Obstet Ginecol 2006;71(5):307-12.

2. Rotman M, Aziz H, Eifel P. Irradiation of pelvic and paraortic nodes in carcinoma of the cervix. Semin Radiat Oncol 1994;4(1):23-9.

3. Haie C, Pejovic MH, Gerbaulet A, Horiot J, Purquier $\mathrm{H}$, Delouche $\mathrm{J}$, et al. Is prophylactic para-aortic irradiation worthwhile in the treatment of advanced cervical carcinoma? Results of a controlled clinical trial of the EORTC radiotherapy group. Radiother Oncol 1998;11(2):101-12.
4. Grigsby PW, Heydon K, Mutch DG, Kim RY, Eifel P. Long-term follow-up of RTOG 92-10: cervical cancer with positive para-aortic lymph nodes. Int J Radiat Oncol Biol Phys 2001;51(4):982-7.

5. Ballon SC, Berman ML, Lagasse LA, Petrilli ES, Castaldo TW. Survival after extraperitoneal pelvic and paraaortic lymphadenectomy and radiation therapy in cervical carcinoma. Obstet Gynecol 1981;57(1):90-5.

6. Weiser EB, Bundy BN, Hoskins WJ, Heller PB, Whittington RR, DiSaia PJ, et al. Extraperitoneal versus transperitoneal selective paraortic lymphadenectomy in the pretreatment surgical staging of advanced cervical carcinoma (a GOG study). Gynecol Oncol 1989;33(3):283-9.

7. Sher ME, Bauert J. Radiation induced enteropathy. Am J Gastroenterol 1990;85(2):121-8.

8. Coia LR, Myerson RJ, Tepper JE. Late effects of radiation therapy on gastrointestinal tract. Int $\mathrm{J}$ Radiat Oncol Biol Phys 1995;31(5):1213-36.

9. Small W, Woloschak G. Radiation Toxicity. A practical guide. Springer-Science New York, 2006.

10. Radiation Therapy Oncology Group (RTOG). Cooperative Group Common Toxicity Criteria: Hallado en: http:/www.rtog.org/members/toxicity/tox.html

11. Bese NS, Hendry J, Jeremic B. Effects of overall treatment time due unplanned interruption during radiotherapy of different tumor sites and practical methods of compensation. Int J Radiat Oncol Biol Phys 2007;68(3):654-61. 